\title{
Interior vertices and edges in bargraphs
}

\author{
Toufik Mansour $^{1}$ and Armend Sh. Shabani ${ }^{2}$ \\ ${ }^{1}$ Department of Mathematics, University of Haifa \\ 3498838 Haifa, Israel \\ e-mail: tmansour@univ.haifa.ac.il \\ ${ }^{2}$ Department of Mathematics, University of Prishtina \\ 10000 Prishtinë, Republic of Kosovo \\ e-mail: armend.shabani@uni-pr.edu
}

\begin{abstract}
In this paper, we consider two statistics on bargraphs, which are defined to be lattice paths in the first quadrant, starting at the origin and ending upon first return to the $x$-axis. Each bargraph is represented as a sequence of columns $\pi_{1} \pi_{2} \ldots \pi_{m}$ such that column $k$ contains $\pi_{k}$ cells. First we enumerate interior vertices, where naturally, interior vertex is a vertex that belongs to exactly four cells of bargraphs. Then we enumerate $d$-edges - edges that contain $d$ interior vertices. More precisely, we find the generating function for the number of bargraphs with $n$ cells and $m$ columns according: to interior vertices and according to horizontal (vertical) $d$-edges. In addition we consider several special cases in detail, where we obtain asymptotic results for total number of statistics under consideration.
\end{abstract}

Keywords: Bargraphs, Generating functions, Interior vertices, Interior edges.

2010 Mathematics Subject Classification: 05A18.

\section{Introduction}

Bargraphs are lattice paths in $\mathbb{N}_{0}^{2}$, where $\mathbb{N}_{0}=\{0,1,2, \ldots\}$, starting at the origin and ending with its first return to the $x$-axis that have three types of steps: an up step $u=(0,1)$, a down step $d=(0,-1)$, and a horizontal step $h=(1,0)$. Note that an up step cannot directly follow a down step, and vice versa. Also note that horizontal steps must all lie strictly above the $x$-axis.

Given a bargraph $B$, four vertices $A(x, y), B(x+1, y), C(x+1, y+1), D(x, y+1)$ that lie either along $B$ or within the area it subtends in the first quadrant determine a cell of $B$. The edges 
of $B$ are the sets $\{A, B\},\{A, D\},\{B, C\},\{C, D\}$. Thus edges are either horizontal or vertical lines.

A bargraph can be identified as a sequence of columns $s=s_{1} s_{2} \cdots s_{m}$ such that the $j$-th column (from the left) contains $s_{j}$ cells, where $m$ denotes the number of horizontal steps of the bargraph. We denote the set of all bargraphs with $n$ cells and $m$ columns by $C_{n, m}$. Bargraphs with $n$ cells and $m$ columns are in a one-to-one correspondence with compositions of $n$ with exactly $m$ parts, thus $\left|C_{n, m}\right|=\left(\begin{array}{c}n-1 \\ m-1\end{array}\right)$ (see [11], p.2).

The origin of bargraphs can be traced back to [1], where it was presented a solution of a linear solid-on-solid (SOS) model, by observing that the problem of SOS walks that do not touch the surface other than last time is equivalent to the problem of enumerating bargraph polygons according to perimeter and area.

Bargraphs, also referred to as wall polyominoes [2] or skylines [3], have recently been studied from different point of views and connections to different fields have appeared. Prellberg and Brak [4] and Feretić [5] proved some fundamental results on bargraph by establishing a generating function in two variables $x$ and $y$ according to the number of horizontal and up steps, respectively. Other enumerative results related to bargraphs were found by Blecher et al., where bargraphs were enumerated according to statistics: levels [6], peaks [7], descents [8] and walls [9]. Deutsch and Elizalde [10] studied bargraphs as Motzkin paths without peaks or valleys. Therein by using the recursive structure of Motzkin paths, bargraphs were enumerated with respect to several statistics (height of the first column, double rises, double falls, corners, etc).

Some recent results in relation to bargraphs appear in $[13,14,17]$ where the authors studied several statistics on bargraphs such as the area and up step on set partitions, border and tangent cells, corners in compositions and set partitions presented as bargraph.

Recently, in [18], the author counted number interior vertices in set partitions which presented as bargraphs, and here we extend it to counting to all bargraphs.

We refer to [15] for statistics on permutations of length $n$ represented geometrically as bargraphs having the same number of horizontal steps.

For results involving enumeration of integer partitions according to the number of corners in their corresponding Ferrers diagrams see [12].

Bargraphs also have connections to statistical physics where they represent frequency diagrams and have been used to model polymers $[1,16]$.

In this paper we will consider new statistics on bargraphs.

The organization of this paper is as follows. In the next section, we introduce new statistics on bargraphs. In the third section, we find the generating function for the bargraphs with $n$ cells and $m$ columns according to the interior vertices (the concepts in this paragraph will be defined in the next section). From this we establish an explicit formula for the total number of interior vertices in all bargraphs with $n$ cells. In the sections 4 and 5 we enumerate bargraphs with $n$ cells and $m$ columns according to number of horizontal d edges and vertical d-edges, respectively. In both those sections we consider some special cases where we find asymptotic formula for the total number of horizontal (vertical) $d$-edges, with $d \in\{0,1,2\}$. 


\section{Preliminaries}

A vertex is called an interior vertex if it is adjacent to exactly four different cells of bargraph $B$, otherwise it is called a boundary vertex. In the Figure 1, it is given the bargraph $B=$ 235243164231 and its interior vertices. Let $\operatorname{Int}_{v}(B)$ denote the set of interior vertices of a given bargraph $B$. Further define $i n t_{v}(B):=\left|\operatorname{Int}_{v}(B)\right|$. For the given bargraph in Figure 1 we have that

$$
\operatorname{Int}_{v}(B)=\{(1,1),(2,1),(2,2),(3,1),(4,1),(5,1),(5,2),(8,1),(8,2),(8,3),(9,1),(9,2)\},
$$

thus we have that $\operatorname{int}_{v}(B)=12$.

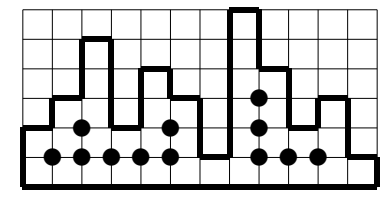

Figure 1. The bargraph $B=235243164231$ and its interior vertices

A horizontal/vertical edge of a bargraph is called a d-h-edgeld-v-edge if it is formed from $d$ interior vertices. Let $E H \operatorname{Int}_{d}(B) / E V \operatorname{Int}_{d}(B)$ denote the set of horizontal/vertical edges formed from $d$ interior vertices. Further define $\operatorname{ehint}_{d}(B):=\left|E_{H} \operatorname{Int}_{d}(B)\right|$ and $\operatorname{evint}_{d}(B):=1$ $E V \operatorname{Int}_{d}(B) \mid$. For the given bargraph in Figure 2 we have that $\operatorname{ehint}_{0}(B)=15, \operatorname{evint}_{0}(B)=12$, $\operatorname{ehint}_{1}(B)=4$, evint $_{1}(B)=10, \operatorname{ehint}_{2}(B)=7$ and evint $_{2}(B)=4$. Clearly,

$$
\sum_{d=0}^{3} \operatorname{ehint}_{d}(B)=\sum_{d=0}^{3} \operatorname{evint}_{d}(B)=n+m
$$

for any bargraph of $n$ with $m$ columns.

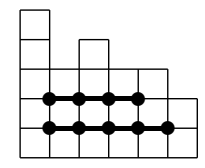

Figure 2. The bargraph 534332 and its 2-edges.

\section{Interior vertices}

Let $I(x, y)=I(x, y ; q)$ (respectively, $\left.I_{a}(x, y)=I_{a}(x, y ; q)\right)$ be the generating function for the number of bargraphs with $n$ cells and $m$ columns (respectively, such that the length of the first column is $a$ ) according to the number of interior vertices, namely,

$$
\begin{aligned}
I(x, y) & =\sum_{n \geq 0} \sum_{m=0}^{n} x^{n} y^{m} \sum_{B \in \mathcal{C}(n, m)} q^{i n t_{v}(B)}, \\
I_{a}(x, y) & =\sum_{n \geq a} \sum_{m=1}^{n} x^{n} y^{m} \sum_{a B^{\prime} \in \mathcal{C}(n, m)} q^{\text {int }_{v}\left(a B^{\prime}\right)} .
\end{aligned}
$$


By definitions, we have

$$
I_{a}(x, y)=x^{a} y+x^{a} y \sum_{b=1}^{a} q^{b-1} I_{b}(x, y)+x^{a} y q^{a-1} \sum_{b \geq a+1} I_{b}(x, y),
$$

which is equivalent to

$$
I_{a}(x, y)=x^{a} y\left(1-q^{a-1}\right)+x^{a} y \sum_{b=1}^{a}\left(q^{b-1}-q^{a-1}\right) I_{b}(x, y)+x^{a} y q^{a-1} I(x, y) .
$$

Define

$$
I(x, y \mid u)=I(x, y ; q, u)=1+\sum_{a \geq 1} I_{a}(x, y) u^{a} .
$$

Clearly, $I(x, y \mid 1)=I(x, y)$. By multiplying (1) by $u^{a}$ and summing over $a \geq 1$, we obtain

$$
I(x, y \mid u)-1=\frac{x y u}{1-x u}+\frac{x y u(1-q)}{q(1-x u)(1-q x u)}(I(x, y \mid q x u)-1)+\frac{x y u}{1-q x u}(I(x, y)-1) .
$$

Let $|x|,|q|,|y|,|u|<1$. By iterating last equation infinitely many times, we obtain

$$
I(x, y \mid u)=1+\sum_{j \geq 0} \frac{(1-q)^{j} q^{\left(\begin{array}{c}
j \\
2
\end{array}\right)} x^{\left(\begin{array}{c}
j+2 \\
2
\end{array}\right)} y^{j+1} u^{j+1}\left(q^{j}(1-q) x^{j+1} u+\left(1-q^{j} x^{j+1} u\right) I(x, y)\right)}{\prod_{i=0}^{j}\left(1-q^{i} x^{i+1} u\right)\left(1-q^{i+1} x^{i+1} u\right)}
$$

By setting $u=1$, and using $I(x, y ; 1)=I(x, y)$, we can state the following result.

Theorem 3.1. We have

$$
I(x, y ; q)=\frac{1+\sum_{j \geq 1} \frac{(1-q)^{j} q^{\left(\begin{array}{l}
j \\
2
\end{array}\right) x} x^{\left(\begin{array}{c}
j+1 \\
2
\end{array}\right)+j} y^{j}}{\prod_{i=0}^{j-1}\left(1-q^{i} x^{i+1}\right)\left(1-q^{i+1} x^{i+1}\right)}}{1-\sum_{j \geq 0} \frac{\left.\left.(1-q)^{j} q^{j}\right)_{x} x_{2}^{j+2}\right)_{y^{j+1}}}{\left(1-q^{j+1} x^{j+1}\right) \prod_{i=0}^{j-1}\left(1-q^{i} x^{i+1}\right)\left(1-q^{i+1} x^{i+1}\right)}} .
$$

Note that $I(x, y ; 1)=\frac{1}{1-\frac{x y}{1-x}}$, as expected. By differentiating $I(x, y ; q)$ with respect to $q$ and evaluating it at $q=1$, Theorem 3.1 gives

$$
\left.\frac{d}{d q} I(x, y ; q)\right|_{q=1}=\frac{d}{d q} \frac{1+(1-q) x^{2} y}{1-\sum_{j=0}^{1} \frac{\left.(1-q)^{j} q^{(j}\right)_{x}\left({ }^{j+2}\right)_{y^{j+1}}}{\left(1-q^{j+1} x^{j+1}\right) \prod_{i=0}^{j-1}\left(1-q^{i} x^{i+1}\right)\left(1-q^{i+1} x^{i+1}\right)}},
$$

which leads to

$$
\left.\frac{d}{d q} I(x, y ; q)\right|_{q=1}=\frac{y^{2} x^{4}}{\left(1-x^{2}\right)(1-x-x y)^{2}} .
$$

Hence, we have the following result.

Corollary 3.2. The total number of interior vertices in all bargraphs with $n$ cells is given by

$$
\frac{3 n-11}{9} 2^{n-2}+\frac{1}{18}\left(9+(-1)^{n}\right) \text {. }
$$




\section{Horizontal $d$-edges}

Let $H(x, y)=H\left(x, y ; q_{0}, q_{1}, q_{2}\right)$ be the generating function for the number of bargraphs with $n$ cells and $m$ columns according to the number of horizontal $d$-edges, namely,

$$
H(x, y)=\sum_{n \geq 0} \sum_{m=0}^{n} x^{n} y^{m} \sum_{B \in \mathcal{C}(n, m)} \prod_{d=0}^{2} q_{d}^{\text {ehint }_{d}(B)} .
$$

Let $B$ be any nonempty bargraph and let its column lengths be $b_{1}, b_{2}, \cdots b_{m}$. Then $B$ can be decomposed as either $m=1$, or $b_{1}, \ldots, b_{m}, m \geq 2$ or there exits $j$ such that $b_{1}, b_{2}, \ldots, b_{j-1}, m \geq$ 2 and $b_{j}=1$. Thus,

$$
\begin{aligned}
H(x, y) & =1+\frac{q_{0}^{2} x y}{1-q_{0} x}+q_{1}^{2} q_{2}^{-2} \tilde{H}\left(x, q_{2} x y\right)+q_{0}^{2} x y(H(x, y)-1) \\
& +q_{0}^{2} x y\left(q_{1}^{2} q_{2}^{-1} \tilde{H}\left(x, q_{2} x y\right)+\frac{q_{0}^{3} x^{2} y}{1-q_{0} x}\right) H(x, y),
\end{aligned}
$$

where $\tilde{H}(x, y)=H(x, y)-1-\frac{q_{0}^{2} x y}{1-q_{0} x}$ is the generating function for the number of bargraphs with $n$ cells and $m \geq 2$ columns according to the number of horizontal $d$-edges. Hence, we can state the following result.

Theorem 4.1. The generating function $H(x, y)=H\left(x, y ; q_{0}, q_{1}, q_{2}\right)$ satisfies the following recurrence relation

$$
H(x, y)=\frac{1-q_{0}^{2} x y+\frac{q_{0}^{2} x y}{1-q_{0} x}+q_{1}^{2} q_{2}^{-2}\left(H\left(x, q_{2} x y\right)-1-\frac{q_{0}^{2} q_{2} x^{2} y}{1-q_{0} x}\right)}{1-q_{0}^{2} x y-\frac{q_{0}^{5} x^{3} y^{2}}{1-q_{0} x}-q_{0}^{2} q_{1}^{2} q_{2}^{-2} x y\left(H\left(x, q_{2} x y\right)-1-\frac{q_{0}^{2} q_{2} x^{2} y}{1-q_{0} x}\right)} .
$$

From the above theorem, we have

$$
H(x, y)=\frac{1}{q_{0}^{2} x y+\frac{\left(1-q_{0}^{2} x y\right)^{2}-\frac{q_{0}^{4}\left(1+q_{0} x\right) x^{2} y^{2}}{1-q_{0} x}}{1-q_{1}^{2} q_{2}^{-2}+\frac{q_{0}^{2}\left(q_{0}-q_{1}^{2} q_{2}^{-1}\right) x^{2} y}{1-q_{0} x}+q_{1}^{2} q_{2}^{-2} H\left(x, q_{2} x y\right)}},
$$

which leads to an explicit formula for $H(x, y)$ in terms of continued fractions.

Corollary 4.2. We have

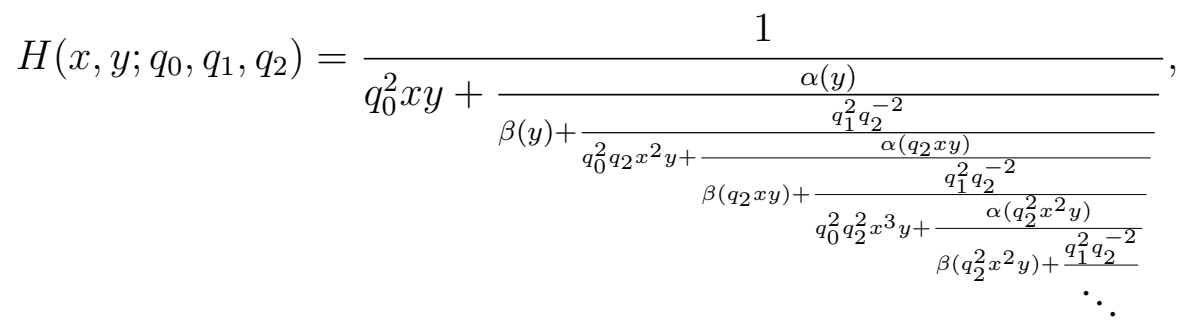

where $\alpha(y)=\left(1-q_{0}^{2} x y\right)^{2}-\frac{q_{0}^{4}\left(1+q_{0} x\right) x^{2} y^{2}}{1-q_{0} x}$ and $\beta(y)=1-q_{1}^{2} q_{2}^{-2}+\frac{q_{0}^{2}\left(q_{0}-q_{1}^{2} q_{2}^{-1}\right) x^{2} y}{1-q_{0} x}$.

Note that by Theorem 4.1 we have that $H(x, y ; 1,1,1)=\frac{H(x, x y ; 1,1,1)}{1-x y H(x, x y ; 1,1,1)}$, which leads to $H(x, y ; 1,1,1)=\frac{1-x}{1-x-x y}$, as expected.

Next, we consider several special cases. 


\subsection{Total number of horizontal 0-edges}

Theorem 4.1 with $q_{0}=q$ and $q_{1}=q_{2}=1$ gives

$$
H(x, y ; q, 1,1)=\frac{\frac{(q-1) q^{2} x^{2} y}{1-q x}+H(x, x y ; q, 1,1)}{1+\frac{(1-q) q^{4} x^{3} y^{2}}{1-q x}-q^{2} x y H(x, x y ; q, 1,1)} .
$$

Let $f(x, y)=\left.\frac{d}{d q} H(x, y ; q, 1,1)\right|_{q=1}$. Then

$$
f(x, y)=\frac{x y\left(x^{5} y^{2}+2 x^{3}(x-1) y-(x-1)^{2}(x-2)\right.}{(1-x-x y)^{2}(1-x)}+\frac{\left(1-x-x^{2} y\right)^{2}}{(1-x-x y)^{2}} f(x, x y),
$$

which implies

$$
f(x, y)=\frac{x y\left(x^{5}(x+1) y^{2}+2 x^{3}\left(x^{3}-1\right) y-(x-2)\left(x^{2}-1\right)\left(x^{3}-1\right)\right)}{\left(1-x^{2}\right)\left(1-x^{3}\right)(1-x-x y)^{2}} .
$$

In particular,

$$
f(x, y)=\frac{\left(2 x^{6}+3 x^{5}+x^{4}-3 x^{3}-2 x^{2}-x+2\right) x}{\left(1-x^{2}\right)\left(1-x^{3}\right)(1-2 x)^{2}} .
$$

Thus, we can state the following result.

Corollary 4.3. Asymptotically, the total number of all horizontal 0-edges over all bargraphs of $n$ is given by $\frac{13 n}{21} 2^{n}$ when $n \rightarrow \infty$.

\subsection{Total number of horizontal 1-edges}

Theorem 4.1 with $q_{1}=q$ and $q_{0}=q_{2}=1$ gives

$$
H(x, y ; 1, q, 1)=\frac{1-x y+\frac{x y}{1-x}+q^{2}\left(H(x, x y ; 1, q, 1)-1-\frac{x^{2} y}{1-x}\right)}{1-x y-\frac{x^{3} y^{2}}{1-x}-q^{2} x y\left(H(x, x y ; 1, q, 1)-1-\frac{x^{2} y}{1-x}\right)} .
$$

Let $f(x, y)=\left.\frac{d}{d q} H(x, y ; 1, q, 1)\right|_{q=1}$. Then

$$
f(x, y)=\frac{2 x^{4} y^{2}\left(1-x-x^{2} y\right)}{(1-x-x y)^{2}(1-x)}+\frac{\left(1-x-x^{2} y\right)^{2}}{(1-x-x y)^{2}} f(x, x y),
$$

which implies

$$
f(x, y)=\frac{\left(1-x^{3}-x^{2}(1+x) y\right) x^{4} y^{2}}{\left(1-x^{2}\right)\left(1-x^{3}\right)(1-x-x y)^{2}} .
$$

In particular,

$$
f(x, y)=\frac{\left(1-x^{2}-2 x^{3}\right) x^{4}}{\left(1-x^{2}\right)\left(1-x^{3}\right)(1-2 x)^{2}} .
$$

Thus, we can state the following result.

Corollary 4.4. Asymptotically, the total number of all horizontal 1-edges over all bargraphs of $n$ is given by $\frac{n}{21} 2^{n}$ when $n \rightarrow \infty$. 


\subsection{Total number of horizontal 2-edges}

Theorem 4.1 with $q_{2}=q$ and $q_{0}=q_{1}=1$ gives

$$
H(x, y ; 1,1, q)=\frac{1+\frac{x^{2} y}{1-x}+q^{-2}\left(H(x, q x y ; 1,1, q)-1-\frac{q x^{2} y}{1-x}\right)}{1-x y-\frac{x^{3} y^{2}}{1-x}-q^{-2} x y\left(H(x, q x y ; 1,1, q)-1-\frac{q x^{2} y}{1-x}\right)} .
$$

Let $f(x, y)=\left.\frac{d}{d q} H(x, y ; 1,1, q)\right|_{q=1}$. Then by using the fact that $H(x, y ; 1,1,1)=\frac{1-x}{1-x-x y}$, we obtain

$$
f(x, y)=\frac{y^{3} x^{6}}{(1-x-x y)^{2}(1-x)}+\frac{\left(1-x-x^{2} y\right)^{2}}{(1-x-x y)^{2}} f(x, x y),
$$

which implies

$$
f(x, y)=\frac{x^{6} y^{3}}{(1-x)\left(1-x^{3}\right)(1-x-x y)^{2}}
$$

In particular,

$$
f(x, y)=\frac{x^{6}}{(1-x)\left(1-x^{3}\right)(1-2 x)^{2}} .
$$

Thus, we can state the following result.

Corollary 4.5. Asymptotically, the total number of all horizontal 2-edges over all bargraphs of $n$ is given by $\frac{n}{28} 2^{n}$ when $n \rightarrow \infty$.

\section{Vertical $d$-edges}

Let $V(x, y)=V\left(x, y ; q_{0}, q_{1}, q_{2}\right)$ be the generating function for the number of bargraphs with $n$ cells and $m$ columns according to the number of vertical $d$-edges, namely,

$$
V(x, y)=\sum_{n \geq 0} \sum_{m=0}^{n} x^{n} y^{m} \sum_{B \in \mathcal{C}(n, m)} \prod_{d=0}^{2} q_{d}^{\text {evint }_{d}(B)} .
$$

Let $\tilde{V}(x, y)=\tilde{V}\left(x, y ; q_{0}, q_{1}, q_{2}\right)$ be the generating function for the number of bargraphs with $n$ cells and $m$ columns according to the number of vertical $d$-edges such that the length of each column is at least 2 .

Let $B$ be any nonempty bargraph and let its column lengths be $b_{1}, b_{2}, \cdots, b_{m}$. Then $B$ can be decomposed as either $b_{1}, \ldots, b_{m} \geq 2, m=b_{1}=1, b_{1}, \ldots, b_{m-1} \geq 2$ and $b_{m}=1$ with $m \geq 2$, $b_{1}=1$ with $m \geq 2$, or there exits $j$ such that $b_{1}, b_{2}, \ldots, b_{j-1} \geq 2$ and $b_{j}=1$ with $2 \leq j \leq m-1$. Thus,

$$
\begin{aligned}
V(x, y) & =\tilde{V}(x, y)+q_{0}^{2} x y+q_{0} x y(\tilde{V}(x, y)-1) \\
& +q_{0} x y(V(x, y)-1)+x y(\tilde{V}(x, y)-1)(V(x, y)-1) .
\end{aligned}
$$

Let $B$ be any nonempty bargraph and let its column lengths be $b_{1}, b_{2}, \cdots, b_{m}$ such that $b_{i} \geq 2$ for all $i=1,2, \ldots, m$. Then $B$ can be decomposed as either $b_{1}, \ldots, b_{m} \geq 3, m=1$ and $b_{1}=2$, 
$b_{1}, \ldots, b_{m-1} \geq 3$ and $b_{m}=2$ with $m \geq 2, b_{1}=2$ with $m \geq 2$, or there exits $j$ such that $b_{1}, b_{2} \ldots, b_{j-1} \geq 3$ and $b_{j}=2$ with $2 \leq j \leq m-1$. Thus,

$$
\begin{aligned}
\tilde{V}(x, y) & =1+\frac{q_{0}^{2}}{q_{2}}\left(\tilde{V}\left(x, q_{2} x y\right)-1\right)+q_{0}^{4} x^{2} y+\frac{q_{1}^{2} q_{0}^{2}}{q_{2}} x^{2} y\left(\tilde{V}\left(x, q_{2} x y\right)-1\right) \\
& +q_{1}^{2} x^{2} y(\tilde{V}(x, y)-1)+\frac{q_{1}^{4}}{q_{0}^{2} q_{2}} x^{2} y\left(\tilde{V}\left(x, q_{2} x y\right)-1\right)(\tilde{V}(x, y)-1) .
\end{aligned}
$$

Hence, we have the following result.

Theorem 5.1. Let $W(x, y)=\tilde{V}(x, y)-1$. Then we have

$$
\begin{aligned}
& V(x, y)=1-q_{0}+\frac{q_{0}+W(x, y)}{1-q_{0} x y-x y W(x, y)}, \\
& W(x, y)=\frac{q_{0}^{2}}{q_{2}} \cdot \frac{q_{0}^{2} q_{2} x^{2} y+\left(1+q_{1}^{2} x^{2} y\right) W\left(x, q_{2} x y\right)}{1-q_{1}^{2} x^{2} y-\frac{q_{1}^{4}}{q_{0}^{2} q_{2}} x^{2} y W\left(x, q_{2} x y\right)} .
\end{aligned}
$$

Note that by finding $W(x, y)$ in terms of $V(x, y)$ and then substituting the expression into the second equation, one can obtain a relation between $V(x, y)$ and $V\left(x, q_{2} x y\right)$. Moreover, Theorem 5.1 with $q_{0}=q_{1}=q_{2}=1$ gives

$$
\begin{aligned}
V(x, y ; 1,1,1) & =\frac{W(x, y ; 1,1,1)}{1-x y-x y W(x, y ; 1,1,1)}, \\
W(x, y ; 1,1,1) & =\frac{x^{2} y+\left(1+x^{2} y\right) W(x, x y ; 1,1,1)}{1-x^{2} y-x^{2} y W(x, x y ; 1,1,1)} .
\end{aligned}
$$

It is not hard to see that $W(x, y ; 1,1,1)=\frac{x^{2} y}{1-x-x^{2} y}$ and $V(x, y ; 1,1,1)=\frac{1-x}{1-x-x y}$. Similarly, as in the previous section, one can show the following result.

Corollary 5.2. The generating function for the total number of all vertical d-edges over all bargraphs of $n$ is given by

$$
\begin{aligned}
\left.\frac{d}{d q_{0}} V\left(x, y ; q_{0}, 1,1\right)\right|_{q_{0}=1} & =\frac{\left(x^{5} y-2 x^{3} y-2 x^{2}-x y+2\right) x y}{\left(1-x^{2}\right)(1-x-x y)^{2}}, \\
\left.\frac{d}{d q_{1}} V\left(x, y ; 1, q_{1}, 1\right)\right|_{q_{1}=1} & =\frac{2 x^{4} y^{2}}{(1-x-x y)^{2}}, \\
\left.\frac{d}{d q_{2}} V\left(x, y ; 1,1, q_{2}\right)\right|_{q_{2}=1} & =\frac{\left(2 x^{2} y+x^{2}+2 x y+x+1\right) x^{6} y^{2}}{\left(1-x^{3}\right)(1+x)(1-x-x y)^{2}}
\end{aligned}
$$

Asymptotically, the total number of all 0-edges (respectively, 1-edges, 2-edges) over all bargraphs of $n$ is given by $\frac{25 n}{48} 2^{n}$ (respectively, $\frac{n}{8} 2^{n}$ and $\frac{13 n}{336} 2^{n}$ ) when $n \rightarrow \infty$.

\section{References}

[1] Owczarek, A. \& Prellberg, T. (1993). Exact solution of the discrete $(1+1)$-dimensional SOS model with field and surface interactions, J. Stat. Phys., 70 (5/6), 1175-1194. 
[2] Duchon, P. (1999). q-Grammars and wall polyominoes, Ann. Comb., 3, 311-321.

[3] Geraschenko, A., An investigation of skyline polynomials, Available online: http:// people.brandeis.edu/\%7egessel/47a/geraschenko.pdf.

[4] Prellberg, T. \& Brak, R. (1995). Critical exponents from nonlinear functional equations for partially directed cluster models, J. Stat. Phys., 78, 701-730.

[5] Feretić, S. (2007). A perimeter enumeration of column-convex polyominoes, Discrete Math. Theor. Comput. Sci., 9, 57-84.

[6] Blecher, A., Brennan, C., \& Knopfmacher, A. (2015). Levels in bargraphs, Ars Math. Contemp., 9, 297-310.

[7] Blecher, A., Brennan, C., \& Knopfmacher, A. (2016). Peaks in bargraphs, Trans. Royal Soc. S. Afr., 71, 97-103.

[8] Blecher, A., Brennan, C., \& Knopfmacher, A. (2016). Combinatorial parameters in bargraphs, Quaest. Math., 39, 619-635.

[9] Blecher, A., Brennan, C., \& Knopfmacher, A. (2017). Walls in bargraphs, Online J. Anal. Combin., 12, 12, 1-11.

[10] Deutsch, E. \& Elizalde, S. (2017). Statistics on bargraphs viewed as cornerless Motzkin paths, Discrete Appl. Math., 221, 54-66.

[11] Heubach, S. \& Mansour, T. (2009). Combinatorics of Compositions and Words, Chapman \& Hall/CRC, Boca Raton.

[12] Blecher, A., Brennan, C., Knopfmacher, A., \& Mansour, T. (2016). Counting corners in partitions, Ramanujan J., 39 (1), 201-224.

[13] Mansour, T. \& Shattuck, M. (2017). Bargraph statistics on words and set partitions, J. Diff. Eq. Appl, 23 (6), 1025-1046.

[14] Mansour, T., Shabani, A. Sh., \& Shattuck, M. (2018). Counting corners in compositions and set partitions presented as bargraphs, J. Diff. Eq. Appl., 24 (6), 849-1022.

[15] Mansour, T. \& Shattuck, M. (2018). Combinatorial parameters on bargraphs of permutations, Trans. Combin., 7 (2), 1-16.

[16] Osborn, J. \& Prellberg, T. (2010). Forcing adsorption of a tethered polymer by pulling, J. Stat. Mech., P09018.

[17] Mansour, T. (2018). Interior vertices in set partitions, Advances in Applied Mathematics, $101,60-69$.

[18] Mansour, T. (2019). Border and tangent cells in bargraphs, Discrete Mathematics Letters, 1, 26-29. 